\title{
Panniculitis Associated with MEK Inhibitor Therapy: An Uncommon Adverse Effect
}

\author{
Miruna Negulescu ${ }^{a} \quad$ Florian Deilhes $^{a}$ Vincent Sibaud $^{a} \quad$ Emilie Tournier $^{b}$ \\ Laurence Lamant $^{\mathrm{b}} \quad$ Serge Boulinguez $^{\mathrm{a}} \quad$ Nicolas Meyer $^{\mathrm{a}, \mathrm{c}}$ \\ ${ }^{a}$ Department of Dermatology, Université Paul Sabatier-Toulouse III et Institut Universitaire \\ du Cancer de Toulouse, Toulouse, France; ${ }^{b}$ Department of Pathology, Université Paul \\ Sabatier-Toulouse III et Institut Universitaire du Cancer de Toulouse, Toulouse, France; \\ 'Inserm UMR 1037-CRCT, Toulouse, France
}

\section{Keywords}

BRAF V600E melanoma · Panniculitis · MEK inhibitors · Cobimetinib · Trametinib

\begin{abstract}
The combination of MEK inhibitor (cobimetinib, trametinib) and BRAF inhibitor (vemurafenib, dabrafenib) is now the first-line treatment in patients with BRAF V600-mutated metastatic melanoma. This association reduces cutaneous adverse events induced by BRAF inhibitors alone, including photosensitivity, hand-foot syndrome, hyperkeratosis, alopecia, skin papillomas, keratoacanthomas, and squamous-cell carcinomas. While panniculitis has exceptionally been reported with BRAF inhibitors, this rare side effect has never been described with the use of MEK inhibitors. We present here the first observation of panniculitis strictly induced by MEK inhibitors. Indeed, 10 days after the initiation of combined treatment with cobimetinib and vemurafenib for metastatic melanoma, our patient developed panniculitis predominantly on the upper and lower extremities. These cutaneous nodules disappeared during cobimetinib intermissions and recurred while the molecule was resumed. Recurrence of cutaneous nodules was observed after initiation of trametinib combined with dabrafenib, and resolved once again with trametinib discontinuation. We believe that clinicians should be aware of this cutaneous adverse event in patients treated with combined therapy, which can lead to unfounded BRAF inhibitor treatment discontinuation and compromise the antitumor
\end{abstract}


response. Our case suggests a class effect linked with the MEK inhibition pharmacodynamic activity. Finally, laboratory investigation and histopathological examination are mandatory to exclude other panniculitis etiologies and subcutaneous metastasis of melanoma.

(C) 2017 The Author(s)

Published by S. Karger AG, Basel

\section{Introduction}

Two phase 3 randomized-controlled trials (COMBI-d and coBRIM) demonstrated that combination of BRAF inhibitors (BRAFi) and MEK inhibitors (MEKi) improves progressionfree survival as compared to BRAFi alone. This combination is now the first-line treatment for patients with BRAF V600-mutated metastatic melanoma [1,2]. Cutaneous adverse effects are significantly reduced with BRAFi and MEKi association including photosensitivity (15\% of patients, coBRIM), hand-foot syndrome (6\% COMBI-d), hyperkeratosis (6\% COMBI-d; $29 \%$ coBRIM), alopecia (5\% COMBI-d; 30\% coBRIM), skin papillomas (1\% COMBI-d), keratoacanthomas ( $8 \%$ coBRIM), and squamous-cell carcinomas (11\% coBRIM) [1-3]. While panniculitis has exceptionally been reported with BRAFi, to the best of our knowledge, this rare side effect has never been described with the use of MEKi [4-10]. Here, we report a patient who developed panniculitis after initiation of combined therapy for metastatic melanoma.

\section{Case Report}

A 48-year-old woman with stage IV BRAF V600E-mutated melanoma started with vemurafenib $960 \mathrm{mg}$ twice daily and cobimetinib $60 \mathrm{mg}$ once daily, 3 weeks a month. Ten days later, she developed tender erythematous nodules and plaques on her upper and lower extremities as well as on the abdomen, clinically suggestive of erythema nodosum-like lesions (Fig. 1). Symmetrical articulation pain and subpyrexia $\left(37.8^{\circ} \mathrm{C}\right)$ were associated with cutaneous condition. Laboratory investigations were strictly normal. Histopathological examination showed no specific perivascular lymphohistiocytic infiltrate in the superficial dermis, but in the deep dermis and hypodermis the infiltrate was composed with a predominance of neutrophils (Fig. 2). In hypodermis, these neutrophils were located in fat lobules and surrounded some capillary walls with fibrinoid necrosis and nuclear debris (Fig. 3). Focal erythrocyte extravasation was seen. These features suggested the diagnosis of lobular panniculitis associated with leukocytoclastic vasculitis. Cutaneous nodules completely disappeared during cobimetinib intermissions and recurred while the molecule was resumed, whereas vemurafenib was maintained. This chronology was a major criterion for cobimetinib accountability. Nonsteroidal anti-inflammatory drugs (ketoprofen $100 \mathrm{mg}$, twice daily) did not prevent occurrence of new lesions, and the treatment was switched due to a lack of improvement with lower doses. Therefore, recurrence of cutaneous nodules was observed after initiation of trametinib, another MEKi combined with dabrafenib, another $\mathrm{BRAFi}$, and resolved once again with trametinib discontinuation. 


\section{Case Reports in Dermatology}

\section{Discussion}

Nearly $50 \%$ of metastatic cutaneous melanomas present a BRAF V600E mutation (substitution of glutamic acid [E] for valine [V] in codon 600) [2]. It induces an activation of the mitogen-activated protein kinase pathway and cellular proliferation. As reported and summarized by Vasquez-Osorio et al. [3], vemurafenib-induced panniculitis seems to affect more women than men (up to 10:9) [7]. The onset of panniculitis in our patient occurred approximately with the same delay (median of 14.5 days) [3]. The clinical presentation of the lesions was similar to other reported cases, with predominance in upper and lower extremities [3-10]. Like our patient, almost all patients developed arthralgia [3-10]. Histopathological examination in previous cases outlined lobular, septal, or mixed (both lobular and septal) panniculitis, in one case associated with vasculitis [3]. Additionally, neutrophil infiltrates usually dominate in the histopathological investigation [3]. This cutaneous effect, already described with vemurafenib, has never been reported with only cobimetinib, or another MEKi. The clinical and histopathological findings are almost similar. The pathomechanism involved in this adverse event has not been elucidated yet. Some authors hypothesized that these symptoms could be linked to a systemic inflammatory reaction to the drug or the melanoma, or to a deregulation of neutrophil migration [3, 7]. However, the paradoxical transactivation of wild-type BRAF, which plays a role in neutrophil migration, is not clearly involved in this precise cutaneous effect because panniculitis has already been reported in combined treatments [7] and is also reported with MEKi (cobimetinib and trametinib) in our case. Most of these cutaneous events associated with arthralgia resolved, thanks to symptomatic treatment. MEKi-induced panniculitis may be more disabling, as in our case, and can lead to definitive treatment discontinuation.

\section{Conclusion}

Deeper investigation is needed to clarify the mechanisms of MEKi-induced panniculitis, which seems to be linked with a class effect of MEKi. Clinicians should be aware of this cutaneous adverse event in patients treated with combined therapy, which can lead to unfounded BRAFi treatment discontinuation and compromise the antitumor response. Finally, laboratory investigation and histopathological examination are mandatory to exclude other panniculitis etiologies and subcutaneous metastasis of melanoma.

\section{Statement of Ethics}

The subject included in this article completed a written consent form before picture taking and biopsy.

\section{Disclosure Statement}

Negulescu M., Deilhes F., Tournier E., Lamant L.: no conflict of interest to declare; Sibaud V.: Roche, GlaxoSmithKline, Pierre Fabre, Merck, Bristol-Myers Squibb, Bayer, Boehringer Ingelheim; Boulinguez S.: Abbvie, MSD, Roche, BMS, Novartis; Meyer N.: Roche, GsK, Novartis, Amgen, BMS, MSD, Pierre Fabre. 


\section{Case Reports in Dermatology}

\section{References}

1 Long GV, Stroyakovskiy D, Gogas H, et al: Dabrafenib and trametinib versus dabrafenib and placebo for Val600 BRAF-mutant melanoma: a multicentre, double-blind, phase 3 randomised controlled trial. Lancet 2015;386:444-451.

-2 Larkin J, Ascierto PA, Dréno B, et al: Combined vemurafenib and cobimetinib in BRAF-mutated melanoma. N Engl J Med 2014;371:1867-1876.

-3 Vázquez-Osorio I, Sánchez-Aguilar MD, García-Rodiño S, Suárez-Peñaranda JM, Aliste C, Vázquez-Veiga $\mathrm{H}$ : Vemurafenib-induced neutrophilic panniculitis: a new case and review of the literature. Am J Dermatopathol 2016;38:E93-E96.

-4 Chaminade A, Conte H, Jouary T, et al: BRAF inhibitors-induced panniculitis: a cutaneous side effect mimicking subcutaneous melanoma metastasis. J Eur Acad Dermatol Venereol 2015;29:392-393.

-5 Choy B, Chou S, Anforth R, Fernández-Peñas P: Panniculitis in patients treated with BRAF inhibitors: a case series. Am J Dermatopathol 2014;36:493-497.

-6 Galliker NA, Murer C, Kamarashev J, Dummer R, Goldinger SM: Clinical observation of panniculitis in two patients with BRAF-mutated metastatic melanoma treated with a combination of a BRAF inhibitor and a MEK inhibitor. Eur J Dermatol 2015;25:177-180.

-7 Mössner R, Zimmer L, Berking C, et al: Erythema nodosum-like lesions during BRAF inhibitor therapy: report on 16 new cases and review of the literature. J Eur Acad Dermatol Venereol 2015;29:17971806.

8 Novoa RA, Honda K, Koon HB, Gerstenblith MR: Vasculitis and panniculitis associated with vemurafenib. J Am Acad Dermatol 2012;67:e271-e272.

-9 Ramani NS, Curry JL, Kapil J, et al: Panniculitis with necrotizing granulomata in a patient on BRAF inhibitor (dabrafenib) therapy for metastatic melanoma. Am J Dermatopathol 2015;37:e96-e99.

10 Sinha R, Edmonds K, Newton-Bishop J, Gore M, Larkin J, Fearfield L: Erythema nodosum-like panniculitis in patients with melanoma treated with vemurafenib. J Clin Oncol 2013;31:e320-e321.

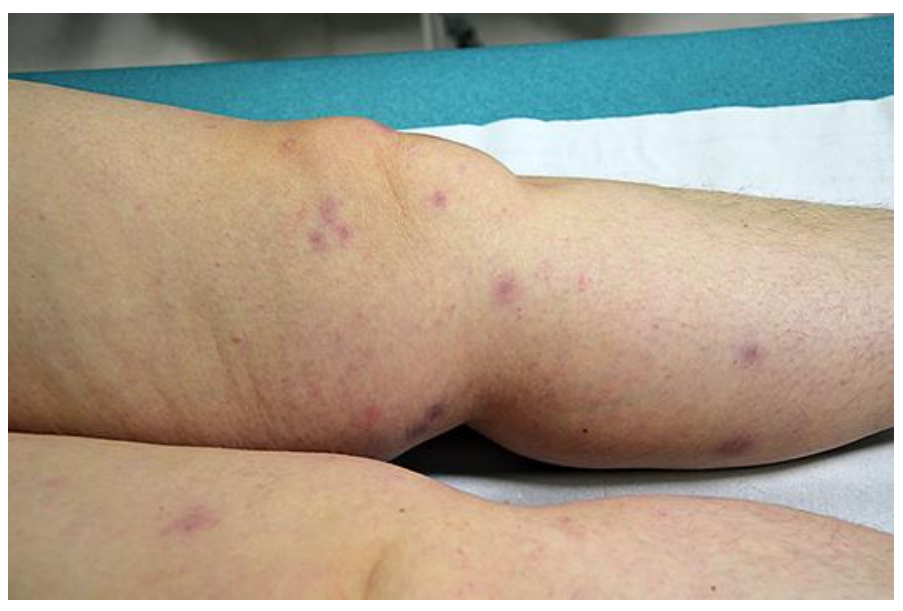

Fig. 1. Purplish nodules on lower extremities. 


\section{Case Reports in Dermatology}

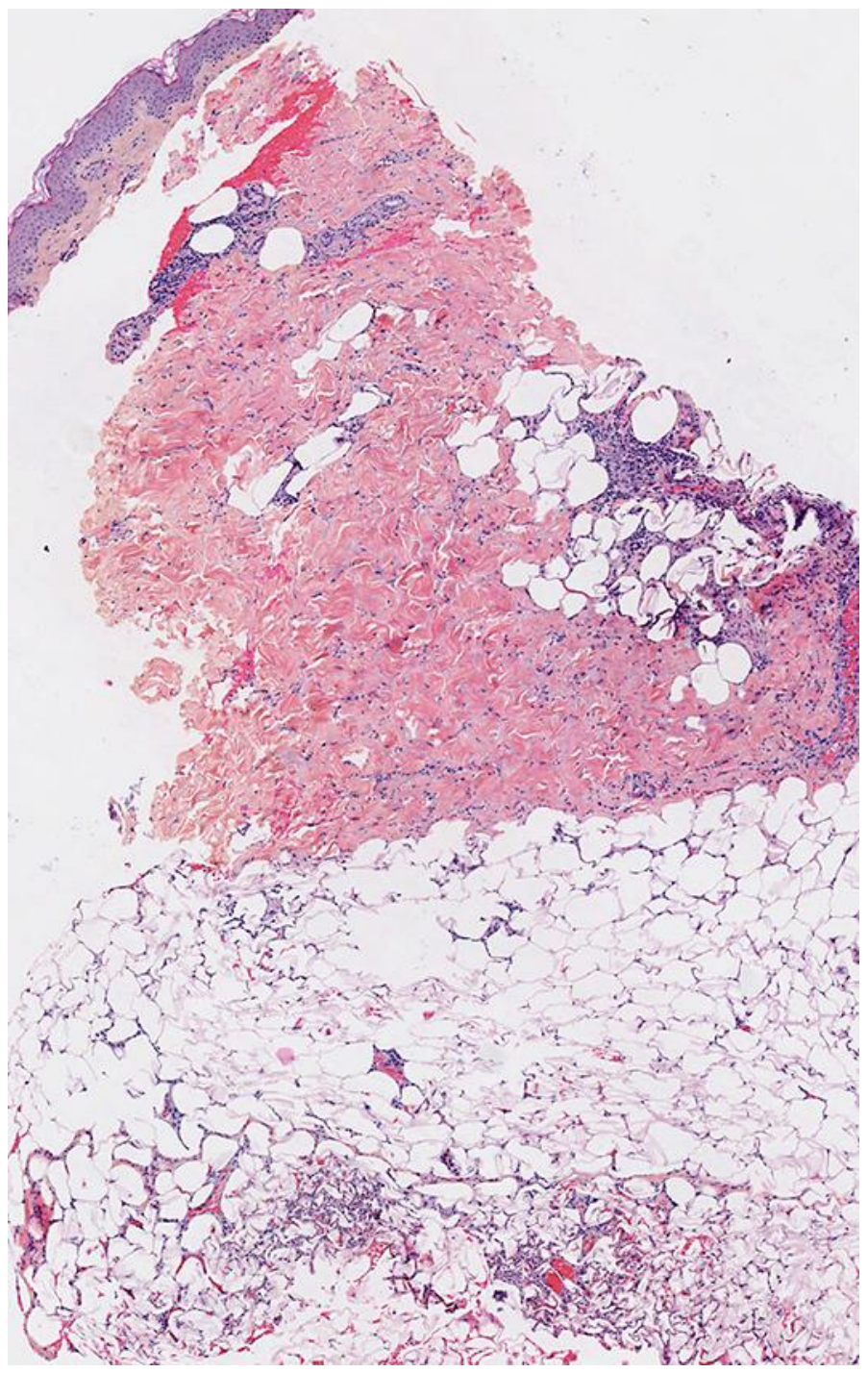

Fig. 2. Hemalun eosin, $\times 50$ magnification. The infiltrate occurred in fat lobules. 


\section{Case Reports in Dermatology}

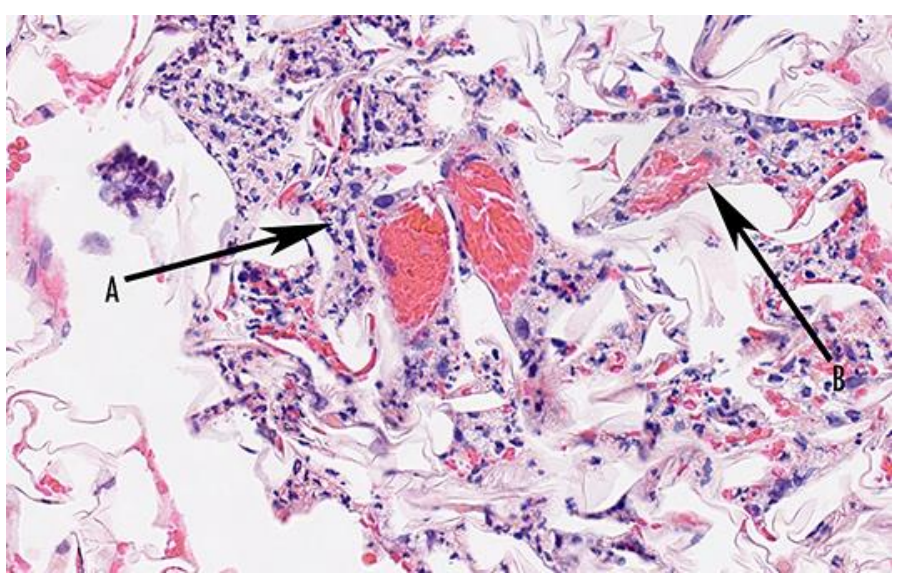

Fig. 3. Hemalun eosin, $\times 400$ magnification. A: Inflammatory compounds (containing polymorphonuclear neutrophils) surrounding hypodermis capillary walls. B: Fibrinoid necrosis of a vascular wall. 